\title{
Contribution à l'étude des Hydroptilidae (Insecta : Trichoptera) de l'Afrique de l'Ouest : le genre Orthotrichia Eaton de la rivière Mouhoun (Burkina Faso)
}

\author{
W. Guendal
}

Mots clés : Trichoptères, Hydroptilidae, Orthotrichia, taxonomie, répartition, rivière Mouhoun, Afrique occidentale.

Le présent travail a été réalisé à partir de récoltes d'insectes aquatiques à la lumière artificielle dans 15 stations du cours axial de la rivière Mouhoun (ex Volta Noire). 12 espèces ont été identifiées. Parmi elles, six sont nouvelles et décrites : Orthotrichia guinkoi n. sp., $O$. dapola n. sp., $O$. prevoti n. sp., $O$. bolyi n. sp., $O$. mussoi n. sp., $O$. cazaubonae n. sp. Certaines espèces sont signalées pour la première fois en Afrique de l'Ouest : O. verbekei Jacquemart, O. nova Marlier, O. alboguttata Jacquemart, $O$. petiti Jacquemart. Deux espèces sont déjà signalées dans la sous-région de l'Afrique occidentale : $O$. straeleni Jacquemart et $O$. aequatoriana Kimmins. Pour chaque espèce, le point est fait sur la répartition le long de la rivière étudiée et éventuellement en Afrique.

Contribution to the study of Hydroptilidae (Insecta : Trichoptera) from West Africa : the genus Orthotrichia Eaton in the river Mouhoun (Burkina Faso)

Keywords : Trichoptera, Hydroptilidae, Orthotrichia, taxonomy, distribution, Mouhoun river, West Africa.

The present study deals with aquatic insects caught at fifteen stations along the river Mouhoun (formerly Black Volta) using artificial light. Twelve species were identified. Among them six are new species and described as : Orthotrichia guinkoi n. sp., $O$. dapola n. sp., $O$. prevoti $\mathrm{n}$. sp., $O$. bolyi n. sp., $O$. mussoi $\mathrm{n}$. sp., $O$. cazaubonae n. sp. Some species are recorded for the first time from Africa : $O$. verbekei Jacquemart, $O$. nova Marlier, $O$. alboguttata Jacquemart, $O$. petiti Jacquemart. Two species are already recorded from Africa : $O$. straeleni Jacquemart and $O$. aequatoriana Kimmins. For every species the distribution along the studied river is given. The distribution in Africa is also given for some species.

\section{Introduction}

La synthèse réalisée par Marlier (1962) sur les Trichoptères d'Afrique mentionne que le genre Orthotrichia est abondamment représenté en Afrique éthiopienne et que l'on connaît huit espèces du genre $O r$ thotrichia d'Afrique. Les travaux antérieurs à cette date sont essentiellement ceux de Mosely (1948), Kimmins (1957b) et Jacquemart (1957). Après la synthèse de Marlier (1962) on doit noter les contributions de Scott (1963), Marlier (1966, 1978), Statzner (1977), Marshall (1979), Wells \& Andersen (1995). Ces investigations ont porté à 16 le nombre d'espèces de Tricho-

\footnotetext{
1. Université de Ouagadougou, Laboratoire d'Écologie, B.P. 7021 Ouagadougou 03, Burkina Faso.
}

ptères du genre Orthotrichia en Afrique, soit le double du précédent.

Le présent travail contribuera à faire connaître six autres espèces, portant ainsi l'inventaire des espèces à 22, presque le triple du nombre recencé en 1962.

\section{Matériel et méthodes}

Le Mouhoun est une rivière permanente du bassin du fleuve Volta qui prend sa source à $530 \mathrm{~m}$ d'altitude sur les hauts-plateaux de Bobo-Dioulasso. Il traverse la savane soudanienne dans le sens sud-nord puis nord-sud dans son cours inférieur et sert de frontière entre le Burkina, le Ghana et la Côte d'Ivoire.

Les échantillons ont été récoltés dans 15 stations situées sur le cours principal du Mouhoun. La figure 1 présente la localisation des stations prospectées.

Les adultes ont été récoltés à la lumière artificielle au début de la nuit, entre 18 et 20 heures. Le système 


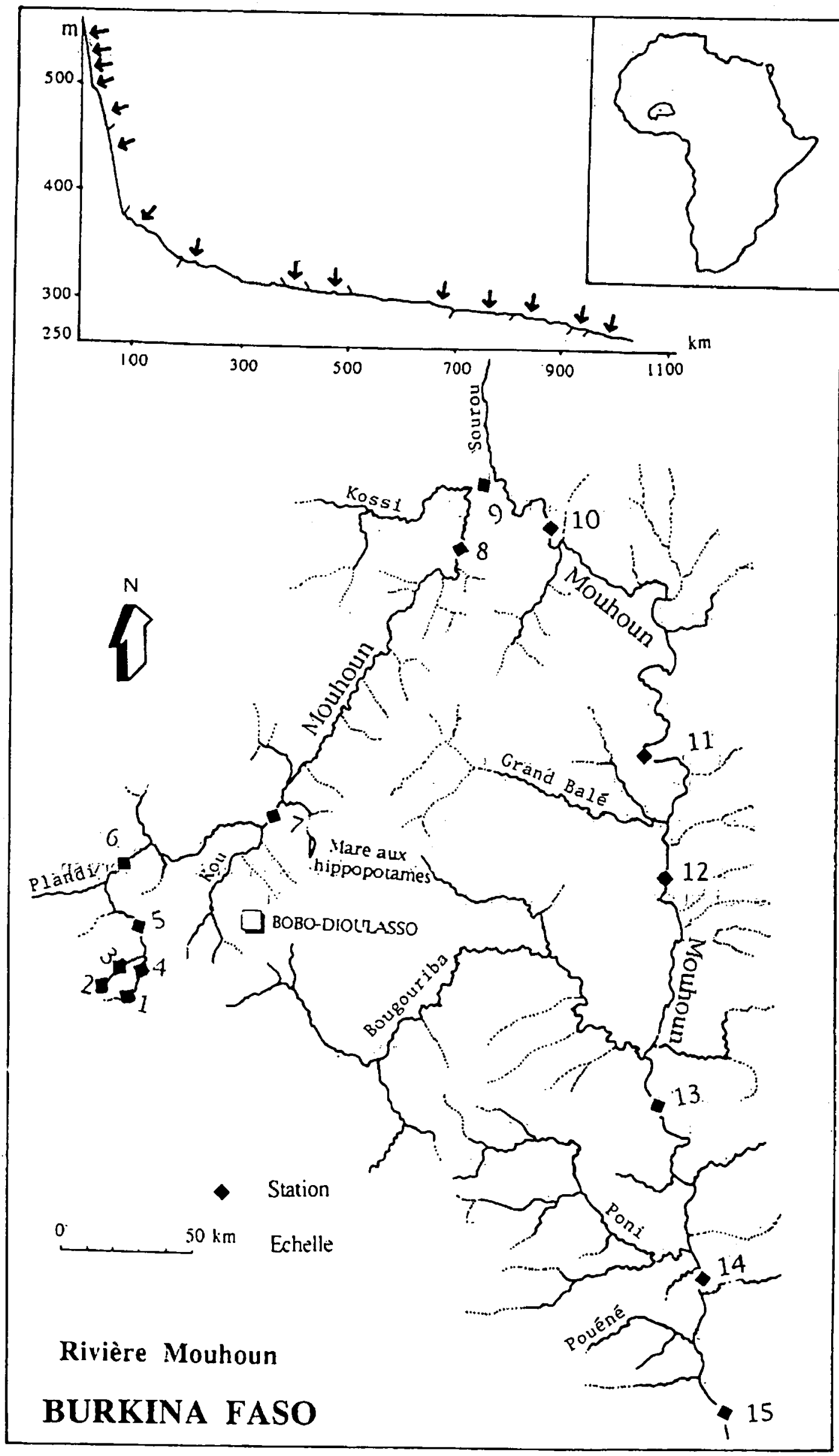

Fig. 1. Localisation des 15 stations prospectées.

Fig. 1. Location of the 15 sites studied. 
de capture est constitué de deux lampes : l'une à ultraviolet et l'autre à gaz. La lampe à ultra-violet est installée sur une toile blanche carrée de $1 \mathrm{mx} 1 \mathrm{~m}$. L'éclat des rayonnements ultra-violets est amplifié par la toile et attire un plus grand nombre d'insectes. La capture est réalisée par aspersion d'alcool à $70^{\circ}$ à l'aide d'une pissette. La lampe à gaz est placée dans un bac contenant de l'eau savonneuse, le savon ayant pour effet de mouiller les ailes des organismes capturés.

L'identification spécifique nécessite la préparation des genitalia. Les individus sont disséqués èt les abdomens sont éclaircis à la potasse puis montés entre lame et lamelle.

\section{Taxonomie et données sur l'habitat et la distribution}

Les stations de récoltes sont réparties dans quatre zones : la zone des sources (stations 1 à 4), le cours supérieur (stations $5,6,7$ ), le cours moyen (stations 8 à 12) et le cours inférieur (stations $13,14,15$ ).

Pour chaque espèce, on donne :

- la liste des stations où elle a été récoltée, les effectifs correspondants et s'il s'agit de larves ; par exemple : St. $1(8)=$ Station $n^{\circ} 1$ (8 adultes récoltés) ;

- un commentaire sur la répartition de l'espèce sur le Mouhoun et sur son habitat ;

- si nécessaire, une information ssur la biologie et la distribution de l'espèce dans d'autres rivières africaines.

Au total, douze espèces ont été identifiées dans le Mouhoun, dont six sont noúvelles pour la Science.

En tenant compte de la structure du neuvième segment abdominal, notamment les plàques dorsale et ventrale, ainsi que celle du phallus, on peut distinguer quatre séries d'espèces :

- La série de Orthotrichia straeleni qui regroupe cinq espèces : Orthotrichia straeleni Jacq., $O$. verbeke $i$ Jacq., O. guinkoi n. sp., $O$. dapola n. sp. et $O$. aequatoriana Kimm. Les caractères distinctifs de ce groupe d'espèces sont constitués par la plaque dorsale triangulaire légèrement inclinée dans la partie distale et la partie latérale droite terminée par une forte et longue soie.

- La série de Orthotrichia alboguttata qui comprend deux espèces : $O$. alboguttata Jacq. et $O$. prevo$t i \mathrm{n}$. sp. La plaque dorsale forme une gouttière caractéristique de cette série d'espèces.

- La série de Orthotrichia nova qui réunit deux espèces : $O$. nova Marlier et $O$. bolyi n. sp. Le rétrécissement progressif de la plaque dorsale du neuvième segment, qui se termine par une extrémité droite, constitue le trait caractéristique de ces espèces.
- La série de Orthotrichia petiti avec trois espèces : $O$. petiti Jacq., $O$. mussoi n. sp. et $O$. cazaubonae n. sp. dont le caractère distinctif est la forme en dôme de la plaque dorsale.

\section{Orthotrichia straeleni Jacquemart, 1957}

Pour cette espèce, nous mentionnons également les récoltes de larves $(\mathrm{L})$.

St. 3 (3) ; St. 4 (16L) ; St. 5 (1) ; St. $6(2)$; St. 7 (16) ; St. 11 (2L); St. 13 (3); St. 14 (1L).

Présente depuis la source jusqu'au cours inférieur, cette espèce n'est pas abondante. Les jeunes stades vivent de préférence sur les feuilles accumulées dans le courant.

La figure 2 représente les genitalia mâles de cette espèce.

Décrite du Kivu au Zaïre (Jacquemart 1957), elle a été signalée également en Ouganda (Kimmins 1957b).

La biologie et l'écologie de $O$. straeleni sont relativement bien connues à travers la description des larves et des nymphes (Jacquemart 1957). C'est la seule espèce, , parmi celles du présent travail, dont les jeunes stades ont été décrits. Elle vit dans la végétation aquatique à l'embouchure des tributaires des lacs Kivu et Edward au Zaïre, les fourreaux des larves et nymphes étant fixés sur les coquilles de Bithynia sp.

Sa distribution dans le Mouhoun est limitée aux zones à couvert végétal important. C'est une espèce commune de la source au cours inférieur. Son apparente raréfaction dans le cours moyen peut être liée au ralentissement du courant et au manque d'oxygène dissous dans ce secteur à méandres.

\section{Orthotrichia verbekei Jacquemart, 1957}

\section{St. 4 (8 mâles)}

Cette espèce a été récoltée dans la zone des sources à Fon et dans le cours moyen à Badala. Les genitalia mâles, représentés sur la figure 3, correspondent à la description originale (Jacquemart 1957).

Décrite du Zaïre (lac Edward), cette espèce n'avait pas été signalée ailleurs sur le continent africain.

\section{Orthotrichia guinkoi n. sp.}

St. 8 (11) ; St. 10 (5); St. 14 (3).

Matériel : L'holotype a été capturé à Zindi le 24/03/1991; 18 paratypes ont été récoltés dans les cours moyen et inférieur du Mouhoun dans les sites de Badala (village proche de Dédougou), de Douroula (village situé entre le Sourou et Boromo) et de Zindi, village au sud de Gaoua, après le confluence de la Bougoriba avec le Mouhoun. 

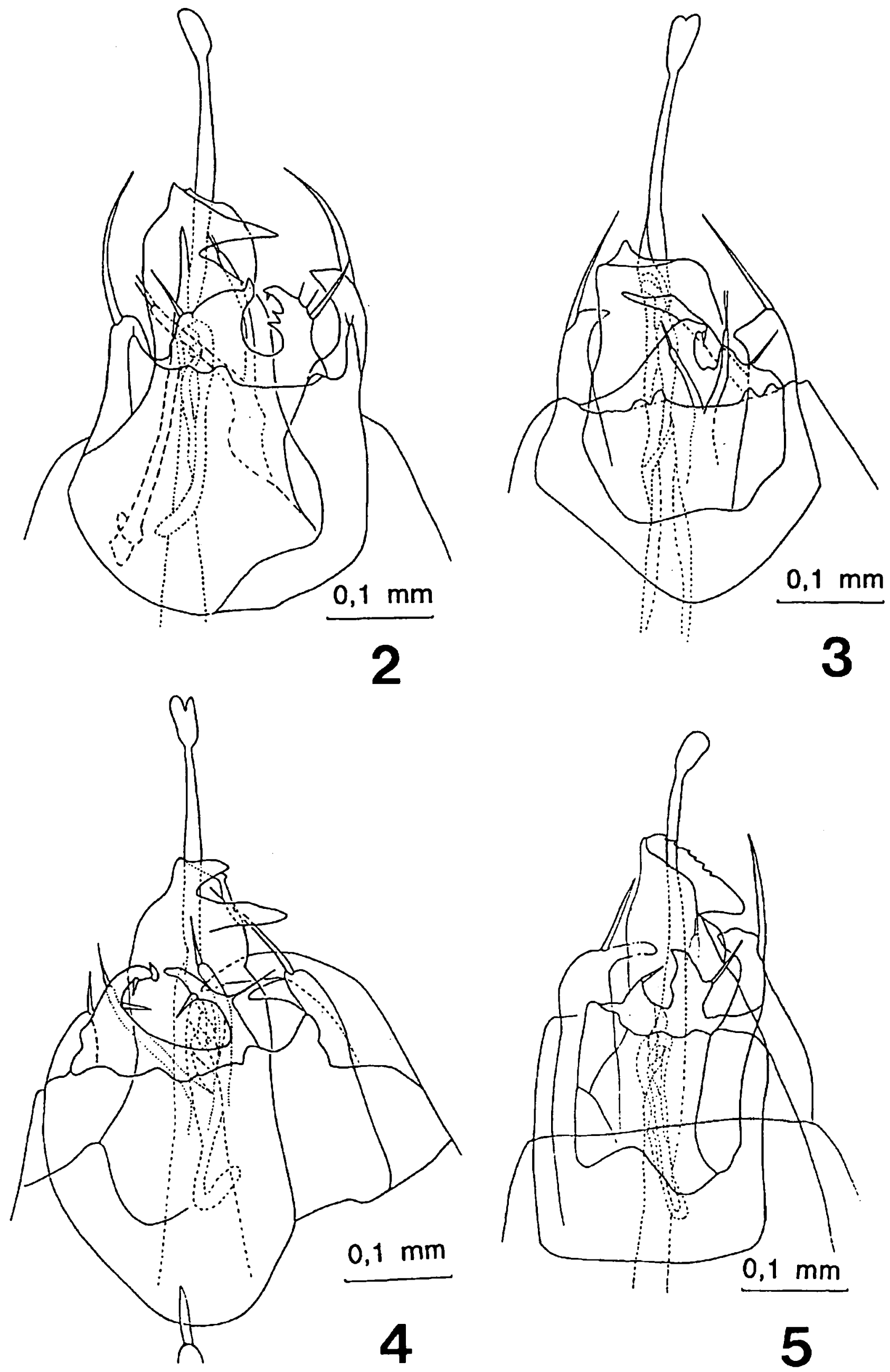

Figs. 2-5. Genitalia mâles (vues ventrales) de : 2. Orthotrichia straeleni Jacq. ; 3. Orthotrichia verbekei Jacq. ; 4. Orthotrichia guinkoi n. sp. ; 5. Orthotrichia dapola n.sp..

Figs. 2-5. Male genitalia (ventral) of : 2. Orthotrichia straeleni Jacq. ; 3. Orthotrichia verbekei Jacq. ; 4. Orthotrichia guinkoi n. sp. ; 5 . Orthotrichia dapola n.sp.. 


\section{Description des genitalia (Fig. 4) :}

La plaque dorsale, large à la base, est rétrécie à l'extrémité ; elle recouvre le phallus dorso-ventralement par le côté gauche ; le bord droit porte deux excroissances à bout aminci, dont la proximale est beaucoup plus longue.

La plaque ventrale est de forme irrégulière et comprend deux lobes asymétriques placés aux bords d'un profond sinus. Le lobe droit est plus allongé que le gauche.

Les pièces latérales, au nombre de deux, sont de taille comparable et placées au même niveau que la plaque ventrale ; l'axe de la pièce gauche, légèrement plus court, est arrondi et muni d'une courte soie ; la pièce droite présente une extrémité plus large avec une excroissance anteapicale pointue et touchant presque le lobe droit de la plaque ventrale ; apicalement, il y a une soie forte et longue.

En vue dorsale, on observe deux pièces latérales longues, à apex effilé, qui convergent vers l'axe dorsoventral jusqu'à se toucher. Le phallus, assez long, porte un filament spiralé.

Espèces voisines: $O$. straeleni Jacquemart, $O$. verbekei Jacquemart, $O$. dapola n. sp.

Etymologie : espèce dédiée au Professeur Dr. Sita Guinko, Université de Ouagadougou, Burkina Faso.

\section{Orthotrichia dapola n. sp.}

St. 13 (2).

Matériel : Holotype et un paratype mâles capturés à Dapola dans le cours inférieur du Mouhoun le 22/03/1992.

\section{Description des genitalia (Fig. 5) :}

La plaque dorsale du neuvième tergite présente une excroissance latérale en forme de triangle dont le bord distal porte des denticulations. La plaque ventrale réduite comprend deux lobes asymétriques dont le plus petit est muni d'une épine terminale et le plus grand porte une épine latérale externe ; les deux lobes sont séparés par un sinus.

Les pièces latérales du huitième segment ont une base large, l'extrémité rétrécie et terminée par une longue et forte soie ; l'appendice se prolonge vers l'axe par une excroissance horizontale.

Le phallus, assez long, présente une zone spiralée.

Espèces voisines : $O$. straeleni Jacquemart, $O$. verbeke $i$ Jacquemart, $O$. guinkoi n. sp. Distribution : cette espèce a été récoltée uniquement dans le cours inférieur du Mouhoun.

Etymologie : Dapola, petit village de la région de Gaoua, Burkina Faso.

\section{Orthotrichia aequatoriana Kimmins, 1957}

St. 1 (2) ; St. 3 (4) ; St. 5 (1) ; St. 6 (1) ; St. 8 (13) ; St. $9(15)$; St. 10 (3) ; St. 11 (1) ; St. 12 (3) ; St. 14 (10) ; St. 15 (3).

Cette espèce est présente depuis la source jusqu'au cours inférieur du Mouhoun. Sans être abondante, elle forme des populations à effectifs moyens.

La figure 6 représente les genitalia mâles de l'espèce..La plaque ventrale du neuvième sternite a une forme caractéristique. L'espèce a été décrite par Kimmins (1957) à partir d'échantillons collectés en Uganda.

Distribution : Espèce commune dans l'ensemble du Mouhoun ; elle devrait avoir une vaste aire de dispersion en Afrique de l'Ouest.

\section{Orthotrichia alboguttata Jacquemart, 1956}

St. 4 (1).

Espèce rare, elle semble limitée à la zone des sources dans le Mouhoun. Elle a été récoltée uniquement à Fon où l'altitude avoisine $500 \mathrm{~m}$.

Les genitalia mâles sont représentés dans la figure 7 . La plaque dorsale forme une gouttière dont une partie couvre le phallus. Décrite du Kivu au Zaïre par Jacquemart (1956) comme habitant la végétation aquatique en présence du courant.

\section{Orthotrichia prevoti n. sp.}

St. 10 (1) ; St. 14 (1) ; St. 15 (2).

Matériel : Holotype récolté à Zindi le 24/03/1992 et paratypes obtenus à Douroula le 2/11/1991 et à Tantama le 23/03/1992.

\section{Description des genitalia (Fig. 8) :}

La plaque dorsale, en forme de voûte à contour irrégulier, ne couvre le phallus que dorso-latéralement.

La plaque ventrale est constituée par des appendices bilobés. En vue ventrale, l'appendice gauche est étroit, l'appendice droit est plus large et fortement émarginé à l'apex.

Les pièces latérales sont représentées par deux éléments recourbés. Tout comme $O$. alboguttata, $O$. prevoti présente au niveau du $8^{\text {emc }}$ segment deux appendices symétriques placés latéralement et terminés par des soies fines:

Le filament spiralé du phallus présente une boucle apicale caractéristique.

Distribution: L'espèce semble être inféodée au cours inférieur dans le Mouhoun. Sa distribution pourrait donc être limitée aux grands cours d'eau.

Etymologie : Espèce dédiée au Dr. Georges Prévôt, Université d'Aix-Marseille III, France. 

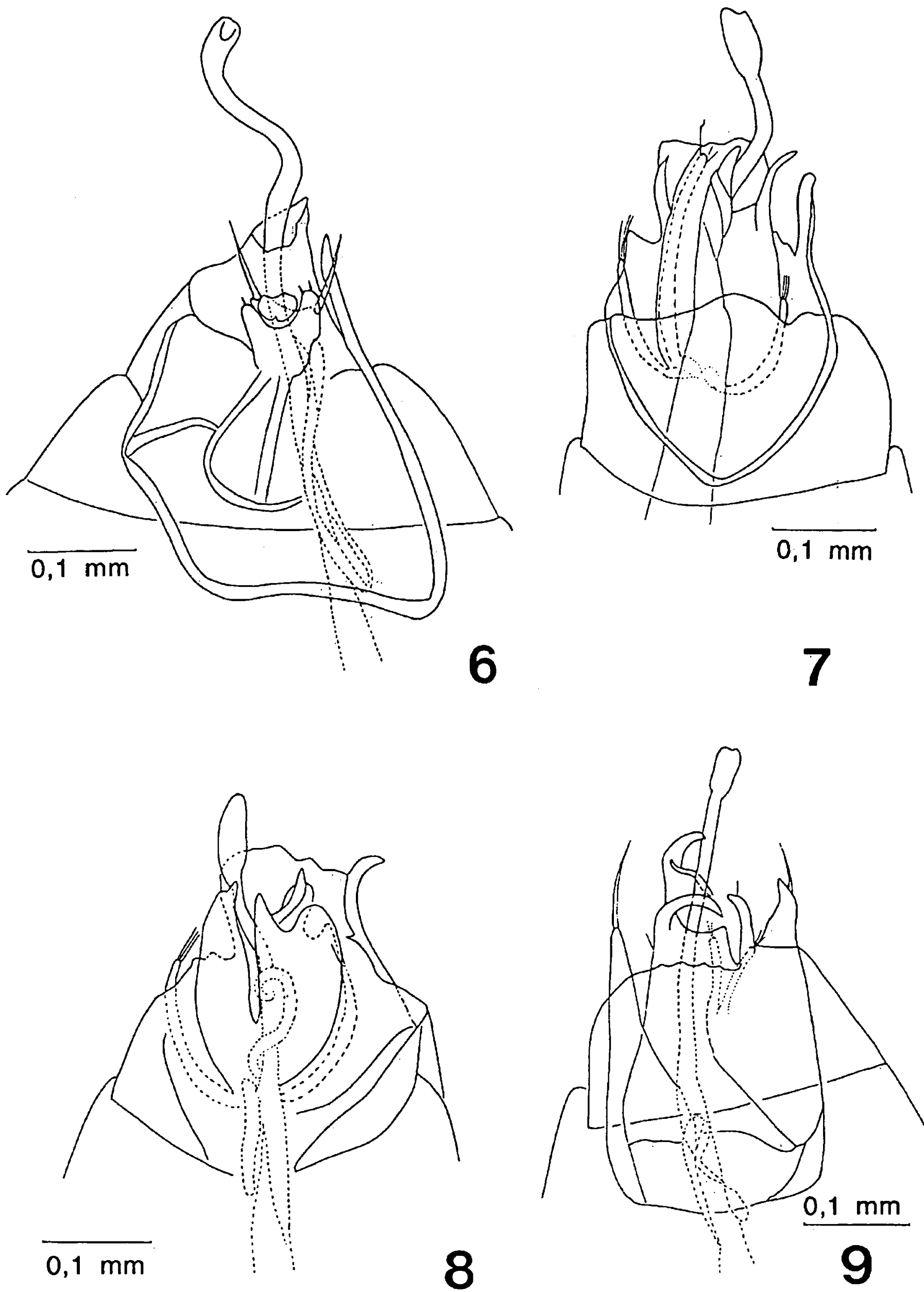

Figs. 6-9. Genitalia mâles (vues ventrales) de : 6. Orthotrichia aequatoriana Jacq. ; 7. Orthotrichia alboguttata Jacq. ; 8. Orthotrichia prevoti n. sp. ; 9. Orthotrichia nova Marlier.

Figs. 6-9. Male genitalia (ventral) of : 6. Oríhotrichia aequatoriana Jacq. ; 7. Orthotrichia alboguttata Jacq. ; 8. Orthotrichia prevoti n. sp. ; 9. Orthotrichia nova Marlier. 


\section{Orthotrichia nova Marlier, 1978}

St. 8 (1) ; St. 9 (1) ; St. 10 (1) ; St. 14 (1).

Présente dans les cours moyen et inférieur (Badala, Kouri, Douroula, Zindi), cette espèce semble préférer les milieux à écoulement modéré.

Les genitalia (Fig. 9) correspondent à la description de Marlier (1978).

Décrite du Mali, sa distribution póurrait comprendre la région soudano-sahélienne.

Orthotrichia bolyi n. sp.

St. 4 (2) ; St. 9 (3) ; St. 10 (1) ; St. 11 (1) ; St. 15 (1).

Matériel : Holotype mâle récolté à Fon dans la zone des sources du Mouhoun le 23/02/1991 ; paratypes mâles capturés dans le cours moyen (Kouri, Douroula, Boromo) et dans le cours inférieur (Tantama).

Description des genitalia (Fig. 10) :

La plaque dorsale du neuvième segment, large à la base, se rétrécit progressivement à partir du tiers puis des deux-tiers de sa longueur et son extrémité est étroite.

La plaque ventrale du $9^{\text {eme }}$ segment comprend deux lobes asymétriques séparés par une profonde échancrure dont les extrémités se touchent presque. Le plus grand lobe présente une excroissance verticale au niveau du bord distal.

Les pièces latérales du $8^{\text {ime }}$ segment, au nombre de 4 , dont 2 sont bifides et 2 autres simples et étroites, sont orientées vers la ligne médiane.

Le phallus, très long, présente un nœud à la hauteur de la plaque dorsale du $9^{\text {eme }}$ segment.

Espèce voisine : $O$. nova Marlier.

Distribution: Cette espèce a été récoltée dans toutes les zones du Mouhoun à l'exception du cours supérieur et ses effectifs sont apparemment très réduits.

Etymologie : Espèce dédiée à Mr. Moussa Boly, Université de Ouagadougou, INSE.

\section{Orthotrichia petiti Jacquemart, 1962}

St. 4 (2).

Cette espèce a été capturée uniquement à Fon, dans la zone des sources du Mouhoun. Décrite par Jacquemart (1962) à partir d'échantillons récoltés dans la région du Katanga au Zaïre ; elle n'avait pas encóre été signalée en Afrique de l'ouest.

Caractéristiques des genitalia (Fig. 11):

Le $9^{\text {ime }}$ tergite est arrondi d'un côté et présente une excroissance de l'autre côté ; il recouvre dorsalement le phallus. Le $9^{\text {eme }}$ sternite forme deux appendices asymétriques, l'appendice gauche étant plus grand et émarginé à l'apex ; le plus petit ayant l'apex assez allongé et étroit ; les deux appendices sont séparés par une petite échancrure oblique.

Les pièces latérales sont caractéristiques du groupe, ce sont deux appendices étroits dont les extrémités arrondies portent au moins deux longues et fortes soies.

On observe aussi une pièce fort allongée, à bout effilé, qui se termine au niveau de la plaque dorsale. Le filament paramère du phallus forme un nœud caractéristique.

Distribution : Espèce sténotope de source qui semble caractéristique des eaux bien oxygénées.

Orthotrichia mussoi n. sp.

St. 8 (105).; St. 9 (4).; St. 10 (35).; St. 11 (1).; St. 13 (1).; St.14 (3):

Matériel : Holótype mâle capturé le 24/03/1992 à Zindi près de Găoua dans le cours inférieur du Mouhoun.

Description des genitalia: (Fig. 12)

La plaque dorsale du $9^{\text {eme }}$ segment est en forme de voûte. Au milieu de la plaque on remarque une portion chitinisée subtrapézoïdale dont l'extrémité distale est rétrécie : le phallus passe entre la plaque proprement dite et l'extrémité proximale de cette partie chitinisée. Le $9^{\text {eme }}$ sternite est une plaque bilobée dont le lobe gauche est en forme de crochet et l'autre tronqué ; les deux lobes sont séparés par une échancrure peu profonde.

Les pièces lạtérales du $8^{\text {eme }}$ segment ont une base large, s'amincissant à l'extrémité et se terminant par 4 longues et fortès soies. Deux autres formations latérales fortement chitinisées constituent une grande plaque dont la base èst située dans le $7^{\text {eme }}$ segment ; l'une de ses extrémités effilées se place en oblique sur la plaque dorsale ; une autre extrémité bifide est insérée vers le $8^{\text {eme }}$ segment.

Le phallus est très long et présente un nœud caractéristique.

Espèces voisines : $O$. petiti Jacq., $O$. cazaubonae n. sp. Distribution : Espèce abondante dans le cours moyen et largement répartie dans le cours inférieur, elle est absente des sources et du cours supérieur. Elle devrait être présente dans d'autres grandes rivières ouest-africaines.

Etymologie : Espèce dédiée au Dr. Joseph-Jean Musso, Université d'Aix-Marseille III, France.

\section{Orthotrichia cazaubonae n. sp.}

St. 8 ('1).

Matériel : Holotype mâle de cette espèce apparemment rare, capturé à Badala, village de la localité de Dédou- 

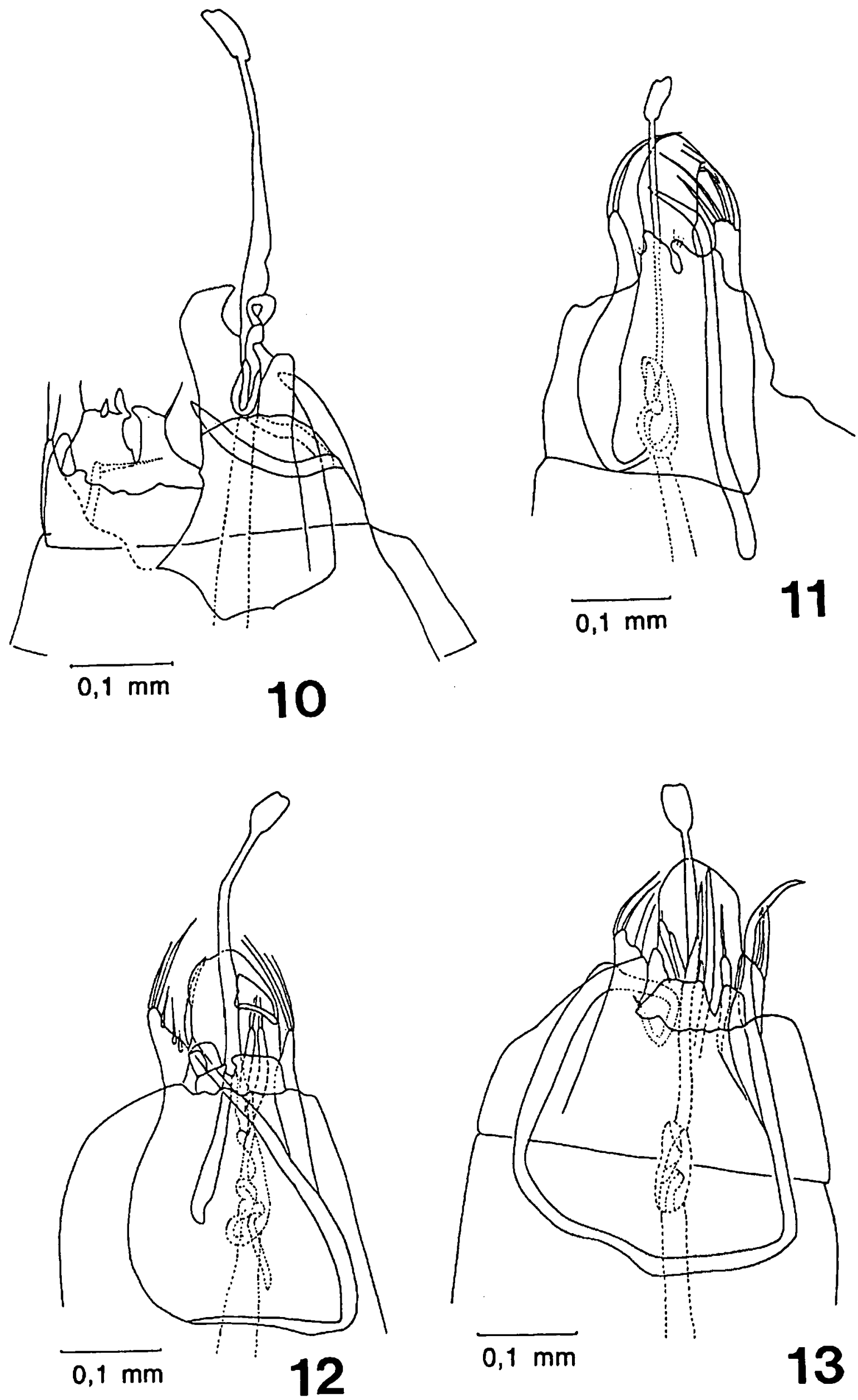

Figs. 10-13. Genitalia mâles (vues ventrales) de : 10 : Orthotrichia bolyi n. sp. ; 11. Orthotrichia petiti Jacq. ; 12. Orthotrichia mussoi n. sp. ; 13. Orthotrichia cazaubonae n. sp.

Figs. 10-13. Male genitalia (ventral) of : 10 : Orthotrichia bolyi n. sp. ; 11. Orthotrichia petiti Jacq. ; 12. Orthotrichia mussoi n. sp. ; 13. Orthotrichia cazaubonae n. sp. 
gou, sur le cours moyen du Mouhoun le 3/11/1991.

\section{Caractéristiques des genitalia (Fig. 13)}

La plaque tergale du $9^{\text {eme }}$ segment forme une voûte recouvrant le phallus. La plaque sternale du $9^{\mathrm{eme}}$ segment comprend deux lobes tronqués de tailles différentes et séparés par une entaille profonde en V.

Les pièces latérales du $8^{\text {ene }}$ segment sont larges et se terminent par quatre longues soies. Deux autres pièces, fines et fortement chitinisées, représentent les extrémités d'une grande plaque dont la base se trouve dans le $7^{\text {tme }}$ segment ; l'une est tournée vers l'extérieur et l'autre forme une forte boucle. La partie distale du phallus est placée en dessous du $9^{\text {ime }}$ segment. Son paramère forme un nœud court.

Espèces voisines: $O$. petiti Jacq., $O$. mussoi $\mathrm{n}$. sp.

Distribution: Cette espèce est actuellement connue seulement du cours moyen du Mouhoun.

Etymologie : Espèce dédiée au Dr. Arlette Cazaubon, Université d'Aix-Marseille III, France.

\section{Discussion}

Sur le cours axial de la rivière Mouhoun, la distribution des espèces appartenant au genre Orthotrichia présente six modalités, compte tenu de l'information actuellement disponible.

Espèces localisées dans la zone des sources : $O$. petiti, O. alboguttata.

Espèce localisée dans le cours moyen : $O$. cazaubonae n. sp.

Espèces qui trouvent leur préférendum écologique dans le cours moyen et qui sont encore présentes dans le cours inférieur : $O$. guinkoi n. sp., $O$. mussoi n. sp.

Espèces eurytopes représentées par $O$. aequatoriana, $O$. bolyi n. sp. et $O$. straeleni.

Espèces localisées dans le cours inférieur : $O$. dapola n. sp., exclusive de cette zone et $O$. prevoti n. sp., préférente de ce tronçon, mais aussi présente dans le cours moyen.

Ce genre n'est pas encore étudié dans toutes les régions de l'Afrique, au sud du Sahara, comme en témoigne la découverte fréquente de nouvelles espèces.

Deux espèces semblent avoir une distribution afrotropicale. Il s'agit de $O$. straeleni et $O$. aequatoriana.

Les espèces connues d'Afrique centrale et présentes sur le Mouhoun, telles $O$. alboguttata, O. nova, O. pe- titi, $O$. verbekei sont signalées pour la première fois en Afrique de l'ouest. Elles pourraient avoir une grande aire de répartition couvrant l'Afrique tropicale.

Le genre Orthotrichia Eaton n'est pas cantonné sur les massifs montagneux africains. Les espèces décrites par Jacquemart (1957) ont été capturées dans la région des grands lacs de l'Afrique centrale et de l'Est africain, mais leur répartition n'est pas déterminée par l'altitude. En effet, les espèces décrites par Statzner (1977) proviennent d'une zone montagneuse du Zaïre. Dans le cas du Mouhoun, le genre Orthotrichia est faiblement représenté en altitude, étant fréquent à partir de $300 \mathrm{~m}$ d'altitude.

Dépôt de matériel : Les holotypes sont actuellement dans la collection de l'auteur, au laboratoire d'Écologie de l'Université de Ouagadougou. Des paratypes sont déposés au laboratoire d'Entomologie du Museum d'Histoire Naturelle de Paris.

\section{Remerciements}

L'auteur remercie le Dr. L. Botosaneanu, Universiteit van Amsterdam, Pays-Bas, d'avoir relu et corrigé le premier manuscrit.

\section{Travaux cités}

Jacquemart S. 1956. - Trois Orthotrichia nouveaux du lac Kivu (Trichoptera, Hydroptilidae). Bull. Inst. Roy. Sc. nat. Belg., 32 : 9.

Jacquemart S. 1962. - Trois Trichoptères nouveaux d'Afrique Centrale. Bull. Inst. Roy. Sc. Nat. Belg., 34 : 1-11.

Kimmins D.E. 1957. - New and little known species of African Trichoptera. Bull. Br. Mus. Nat. Hist. Entom., 6: 1-37.

Marlier G. 1962. - Genera des Trichoptères de l'Afrique. Ann. Mus. Roy. Afr. Centr., Sér. 8, Zool., 109 : 7-259.

Marlier G. 1966. - Les Trichoptères du Musée de Dundo. Comp. Diam. Angol. Mus. Dundo, 30 : 17-79.

Marlier G. 1978. - Sur une collection de Trichoptères de l'Afrique Occidentale. Rev. Zool. Afr., 92, $2:$ 283-302.

Marlier G. 1986. - Sur une petite collection de Trichoptères du Niger. Bull. Inst. Roy. Sc. Nat. Belg. Entom., 56 : 23-24.

Marshall J.E. 1979. - A review of the genera of the Hydroptilidae (Trichoptera). Bull. Br. Mus. Nat. Hist. Entom., 39 (3) : 135-239.

Morse J.C. 1974. - New caddisflies (Trichoptera) from southern Africa. Journal of the Kansas Entomol. Soc., 47 (3) : 328-344.

Mosely M.E. 1948. - Trichoptera collected by Miss R.H. Mowe at Lake Nyasa. Ann. Mag. N. Hist., 12 (1) : $31-47$.

Scott K.M.F. 1963. - Some new African caddisflies (Trichoptera) from the Western Cape Province. IV : Some Hydroptilidae. Am. S. Afr. Mus., 46, $19: 469-478$.

Statzner B. 1977. - Taxonomische Studien an den Hydroptilidae Imagines aus dem zentralafrikanischen Bergbach Kalengo. 5. Trichoptera. Dtch. Ent. Z. N.F. 25 Heft 4-5 : 393-405.

Wells A. et Anderson T. 1995. - Tanzanian micro-caddisflies (Trichoptera : Hydroptilidae) Tijdschrift voor Entomol., 138 : 143-167. 